\title{
Difference in the Location and Risk Factors of Cerebral Microbleeds According to Ischemic Stroke Subtypes
}

\author{
Bum Joon Kim, ${ }^{\mathrm{a}}$ Youngshin Yoon, ${ }^{\mathrm{b}}$ Hoyon Sohn, ${ }^{\mathrm{b}}$ Dong-Wha Kang, ${ }^{\mathrm{b}}$ Jong S. Kim, ${ }^{\mathrm{b}}$ Sun U. Kwon ${ }^{\mathrm{b}}$ \\ ${ }^{a}$ Department of Neurology, Kyung Hee University Hospital, Seoul, Korea \\ bDepartment of Neurology, Asan Medical Center, University of Ulsan College of Medicine, Seoul, Korea
}

Background and Purpose The location of cerebral microbleeds (CMBs) may differ according to ischemic stroke subtype, and the underlying pathomechanism may differ by their location. Here, we investigated the characteristics of CMBs according to various ischemic stroke subtypes to verify this issue.

Methods Patients with acute ischemic stroke were consecutively included. The presence of CMBs was determined by gradient echo image sequence. The distribution of CMBs was classified as deep, lobar, or diffuse (both deep and lobar). The prevalence, risk factors, and distribution of CMBs were compared among patients with different stroke subtypes. Factors associated with the distribution of CMBs were investigated.

Results Among the 1033 patients included in this study, ischemic stroke subtypes were classified as large artery atherosclerosis (LAA; $n=432$ ), small vessel occlusion (SV0; $n=304$ ), and cardioembolism (CE; $n=297)$. The prevalence of CMBs was highest in patients with SVO (40.5\%), followed by CE $(33.0 \%)$ and LAA $(24.8 \% ; P<0.001)$. The locations of CMBs was different according to subtype $(P=0.004)$. CE [odds ratio $(\mathrm{OR})=1.85(1.02-3.34) ; P=0.042]$ and the use of antithrombotics [OR=1.80 (1.10-2.94); $P=0.019]$ were associated with lobar CMBs, and old age [OR=1.02 (1.00-1.04); $P=0.015]$ and hypertension [OR=1.61 (1.08-2.40); $P=0.020$ ] were associated with deep CMBs.

Conclusions CMBs were frequently located in the lobar area in patients with CE. Previous use of antithrombotic agents is associated with lobar CMBs. The pathogenic mechanism of CMB may differ according to ischemic stroke subtype and location.

Keywords Cerebral microbleeds; Classification; Location; Risk factors; Magnetic resonance image

\author{
Correspondence: Sun U. Kwon \\ Department of Neurology, Asan Medical \\ Center, University of Ulsan College of \\ Medicine, 88 Olympic-ro 43-gil, Songpa- \\ gu, Seoul 05505, Korea \\ Tel: +82-2-3010-3960 \\ Fax: +82-2-474-4691 \\ E-mail:sukwon@amc.seoul.kr
}

Received: April 27, 2016

Revised: July 27, 2016

Accepted: August 7, 2016

This study was supported by a grant of the Korean Health Technology R\&D Project, Ministry of Health \& Welfare, Republic of Korea (HI14C1731).

The authors have no financial conflicts of interest.

\section{Introduction}

Cerebral microbleeds (CMBs) are defined as hypointense lesions on $2^{*}$-weighted gradient echo images (GRE) caused by previous microhemorrhages. ' CMBs are regarded as one of the indicators of cerebral small vessel disease, ${ }_{1}^{2,3}$ especially when located in the deep gray matter or infratentorial area. ${ }^{4}$ However, CMBs can also be found in lobar (cortical) areas. Risk factors associated with CMBs in the lobar area are different than those associated with deep CMBs. ${ }^{5,6}$ A different pathomechanism of cortical microangiopathy by amyloid deposition (i.e., cerebral amyloid angiopathy) is regarded as the major cause of lobar CMB ${ }^{5,7}$ In addition, lobar CMBs are also predominantly observed in patients with a cardioembolic source of septic emboli, which may cause small mycotic aneurysms in their cerebral arteries. ${ }^{8}$

The prevalence of CMBs in stroke patients differs according to the subtype of ischemic stroke. The prevalence has been found to be highest in patients with small lacunar infarctions; ${ }^{9-11}$ however, 
CMBs in other subtypes of stroke are also common. ${ }^{12,13}$ In fact, up to one-third of cardioembolic stroke (CE) cases demonstrate CMBs. ${ }^{14-16}$ Considering that patients with CE require long-term anticoagulation, and that anticoagulation increases the risk of intracerebral hemorrhage, especially in patients with multiple $\mathrm{CMBs}$, understanding the pathomechanism of CMBs according to ischemic stroke subtype or location may be important in determining the treatment strategy for secondary stroke prevention.

In our current study, we investigated the risk factors and locations of CMBs in patients with different ischemic stroke subtypes, and the risk factors for CMBs in different locations.

\section{Methods}

\section{Patients}

Ischemic stroke patients who were admitted within 1 week of stroke onset to the stroke center at Asan Medical Center (Seoul, Korea) between January 2007 and January 2010 were retrospectively reviewed from a prospectively collected database. Among these cases, patients classified as having large artery atherosclerosis (LAA), small vessel occlusion (SVO), or CE were consecutively enrolled after excluding other etiologies. The ischemic stroke subtypes in this population were classified in accordance with the TOAST (Trial of ORG 10172 in Acute Stroke Treatment) system. ${ }^{17}$ Electrocardiography was performed in all patients for classification. Holter monitoring, and transthoracic and/or transesophageal echocardiography were performed in selected patients who (1) had a history of heart disease or arrhythmia on electrocardiography, (2) were young ( $<50$ years of age), or (3) had any cortical lesion on diffusion-weighted image without evidence of the embolic source on magnetic resonance angiography. Patients classified as undetermined or other determined etiology and patients with poor quality image that were not amenable to analysis were excluded from the study. The Institutional Review Board of 00 Medical Center approved this study. The requirement for obtaining informed consent was waived because of the retrospective nature of the analysis.

\section{Clinical data}

The demographic characteristics of the study patients, including their vascular risk factors and previous use of antithrombotic agents (antiplatelet agent and anticoagulants) were collected from medical records. The risk factors included hypertension (defined as receiving medications for hypertension or blood pressure $>140 / 90 \mathrm{mmHg}$ on repeated measurements), diabetes mellitus (defined as receiving medications for diabetes mellitus, fasting blood sugar $\geq 126 \mathrm{mg} / \mathrm{dL}$ or $\mathrm{HbA} 1 \mathrm{c} \geq 6.5 \%$, or a casual plasma glucose $>200 \mathrm{mg} / \mathrm{dL}$ ), previous heart disease, hypercholesterol- emia (defined as receiving cholesterol-reducing agents or an overnight fasting cholesterol level $\geq 240 \mathrm{mg} / \mathrm{dL}$, $\geq 200 \mathrm{mg} / \mathrm{dL}$ triglycerides, or Low density lipoprotein (LDL) cholesterol $\geq 160$ $\mathrm{mg} / \mathrm{dL}$ ), and current cigarette smoking. ${ }^{18}$

\section{Imaging protocol and analysis}

MRI scans were obtained within one week of stroke onset using either a $1.5 \mathrm{~T}$ or $3.0 \mathrm{~T}$ MR imaging unit. All the enrolled patients underwent diffusion-weighted image, GRE, and magnetic resonance angiography. The common parameters for diffusionweighted image were a slice thickness of $5 \mathrm{~mm}$, an inter-slice gap of $2 \mathrm{~mm}, 20$ axial slices, and a field of view of $250 \mathrm{~mm}$. The MRI parameters for GRE were a slice of thickness of $5 \mathrm{~mm}$, an inter-slice gap of $2 \mathrm{~mm}, 20$ axial slices, a field of view of 250 $\mathrm{mm}$, a 400-ms repetition time, 30-ms echo time, $20^{\circ}$ flip angle, and a $256 \times 192$ matrix. Common magnetic resonance angiography parameters included a flip angle of $20^{\circ}$, a $512 \times 512$ matrix, and a field of view of $250 \mathrm{~mm}$.

CMBs were defined as lesions with homogeneous round signal loss and a diameter $\leq 5 \mathrm{~mm}$ on GRE. ${ }^{9}$ However, hypointense lesions within the subarachnoid space and areas of symmetrical hypointensity in the globus pallidus on GRE were considered likely to represent adjacent pial blood vessels and calcifications, respectively. ${ }^{9}$ The locations of the CMBs were classified as lobar, deep, or diffuse. CMBs located at the cortico-subcortical area of each lobe (i.e., frontal, parietal, temporal, or occipital) were regarded as lobar CMBs. CMBs in the basal ganglia, thalamus, brainstem, and cerebellum were categorized as deep CMBs. Multiple CMBs that were distributed throughout both the lobar and deep areas were defined as diffuse CMBs. If at least one CMB existed at lobar area or deep area, the patient was regarded to have any lobar or any deep CMB. The number of CMBs was counted separately in each area. Classification of stroke subtype and detection of the CMBs were performed by two independent neurologists (Y.Y and H.S) who were blind to the clinical data, and any discrepancies were reevaluated at a consensus meeting.

\section{Data analysis}

The CMB prevalence was compared between patients with different stroke subtypes. The risk factors for $\mathrm{CMBs}$ were also compared. Pearson chi-square test, student t test, or MannWhitney test was appropriately used. In addition, multivariable logistic regression analysis was performed to estimate the independent contributions of various factors on the presence of CMBs in different stroke subtypes and locations. Variables were selected for entry into the model based on the results of univariable analysis $(P \leq 0.2)$; SVO group: age, hypertension and previous stroke history, LAA group: hypertension and previous stroke 
history, CE group: hypertension and previous use of antithrombotic agents were entered. For locations, factors associated with any lobar, lobar, any deep and deep CMBs were investigated. The odds ratio (OR) and 95\% confidence interval were also determined. The concordance rates between the two investigators were evaluated. A 2-tailed $P$ value $<0.05$ was considered statistically significant. SPSS for Windows was used for these analyses (version 18.0; SPSS Inc., Chicago, IL, USA).

\section{Results}

\section{Study population}

During the study period, 1,357 patients within 7 days from ischemic stroke onset were registered to the database during the study period. Among them, 169 patients with undetermined etiology and 64 patients with other determined etiology were excluded. Of them, 1,124 patients were initially determined to be eligible to participate and were screened. However, 91 patients were excluded due to suboptimal image quality and a final study population of 1,033 patients was included. The ischemic stroke subtypes were LAA $(n=432 ; 41.8 \%)$, SVO $(n=304 ; 29.4 \%)$, and CE $(n=297 ; 28.8 \%$; Figure 1). The demographic features and vascular risk factors were significantly different between patients with different ischemic stroke subtypes.

Compared with LAA or SVO patients, the CE cases were significantly more likely to be elderly. Most of the vascular risk factors, including hypertension, diabetes mellitus, hyperlipidemia, and smoking, were less frequent in CE patients. However, the propor- tion of CE patients who had previously used antithrombotic agents was significantly higher than the other subtypes (Table 1).

\section{Distribution of $\mathrm{CMB}$}

The prevalence and mean number of CMBs were significantly different between the various ischemic stroke subtypes: prevalence, SVO (40.5\%), CE (33.0\%), and LAA (24.8\%; $P<0.001$; Table 1); mean number of CMBs, SVO (mean \pm Standard deviation: 4.65 \pm 7.40$), C E(3.09 \pm 3.49)$, and LAA $(3.12 \pm 3.63 ; P=0.041)$. As indicated in Table 1 and Figure 1, the location of the CMBs also

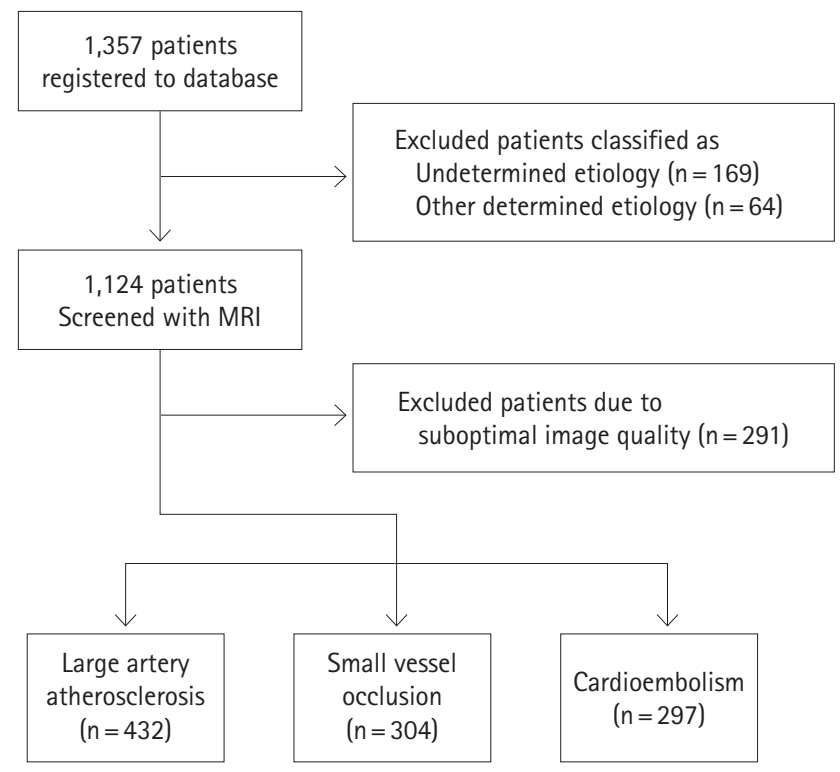

Figure 1. Study flow chart.

Table 1. Baseline characteristics of the study population

\begin{tabular}{|c|c|c|c|c|}
\hline & $\begin{array}{l}\text { Cardioembolism } \\
\qquad(n=297)\end{array}$ & $\begin{array}{l}\text { Small vessel occlusion } \\
\qquad(n=304)\end{array}$ & $\begin{array}{l}\text { Large artery atherosclerosis } \\
\qquad(n=432)\end{array}$ & $P$ \\
\hline Male & $160(53.9)$ & $180(59.2)$ & $283(65.5)$ & 0.006 \\
\hline Age (year) & $70.0[10.3]$ & $64.4[12.3]$ & $65.1[11.2]$ & $<0.001$ \\
\hline Hypertension & $208(70.0)$ & $236(77.6)$ & $336(77.8)$ & 0.034 \\
\hline Diabetes Mellitus & $67(22.6)$ & $103(33.9)$ & $164(38.0)$ & $<0.001$ \\
\hline Hyperlipidemia & $80(26.9)$ & $185(60.9)$ & $244(56.5)$ & $<0.001$ \\
\hline Coronary artery disease & $32(10.8)$ & $15(4.9)$ & $43(10.0)$ & 0.019 \\
\hline Current smoking & $104(35.0)$ & $132(43.4)$ & $203(47.0)$ & 0.005 \\
\hline Previous stroke history & $63(21.2)$ & $75(24.7)$ & $121(28.0)$ & 0.113 \\
\hline Previous use of antithrombotic medication & $176(59.3)$ & $87(28.6)$ & $161(37.3)$ & $<0.001$ \\
\hline Antiplatelet agent & $111(37.4)$ & $87(28.6)$ & $159(36.8)$ & \\
\hline Anticoagulants & $65(21.9)$ & 0 & $2(0.5)$ & \\
\hline The presence of cerebral microbleeds (CMB) & $98(33.0)$ & $123(40.5)$ & $107(24.8)$ & $<0.001$ \\
\hline Presence of any lobar CMB & $63(21.2)$ & $71(23.4)$ & $43(10.0)$ & $<0.001$ \\
\hline Presence of any deep CMB & $71(23.9)$ & $97(31.9)$ & $85(19.7)$ & 0.001 \\
\hline Location of $\mathrm{CMB}$ & & & & 0.004 \\
\hline Lobar & $27(9.1)$ & $26(8.6)$ & $22(5.1)$ & \\
\hline Deep & $35(11.8)$ & $52(17.1)$ & $64(14.8)$ & \\
\hline Diffuse & $36(12.1)$ & 45 (14.8) & $21(4.9)$ & \\
\hline Mean number of $\mathrm{CMB}$ & 3.09 [3.49] & $4.65[7.40]$ & $3.12[3.63]$ & 0.041 \\
\hline
\end{tabular}

Results are expressed as a number (\%) or mean [SD]. 


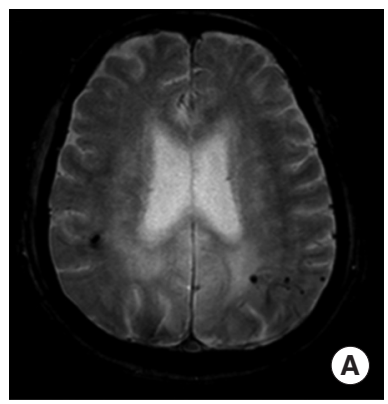

Lobar CMBs

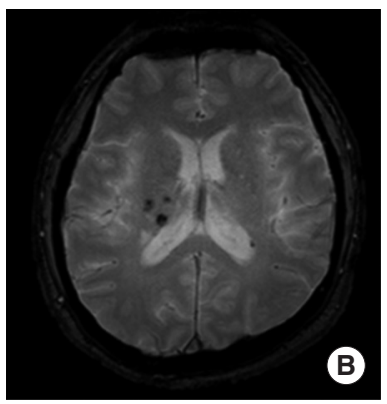

Deep CMBs

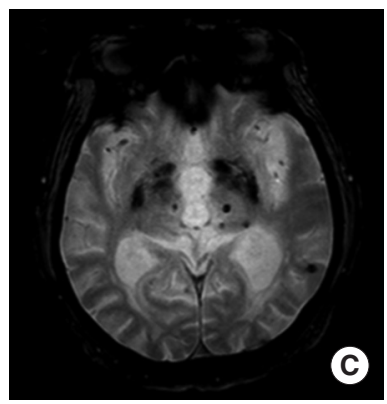

Diffuse CMBs
- Lobar CMBs - Deep CMBs Diffuse CMBs

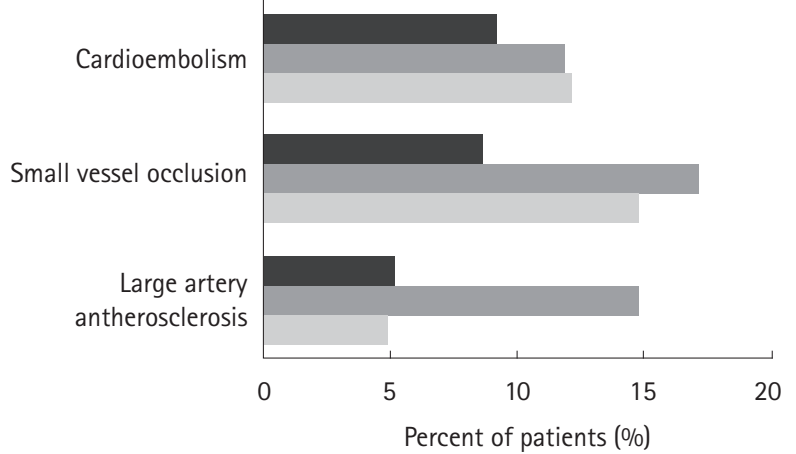

- Any Lobar $C M B=$ Lobar CMB+Diffuse CMB - Any Deep $\mathrm{CMB}=$ Deep $\mathrm{CMB}+$ Diffuse $\mathrm{CMB}$

Figure 2. Location and percentage of patients with cerebral microbleeds (CMBs) in the lobar area compared to that in the deep area in terms of stroke subtypes.

Table 2. Risk factors in patients with and without cerebral microbleeds (CMBs) based on stroke subtype

\begin{tabular}{|c|c|c|c|c|c|c|}
\hline & \multicolumn{2}{|c|}{ Cardioembolism $(n=297)$} & \multicolumn{2}{|c|}{ Small vessel occlusion $(n=304)$} & \multicolumn{2}{|c|}{ Large artery atherosclerosis $(n=432$} \\
\hline & $\begin{array}{l}\mathrm{CMB}+ \\
(\mathrm{n}=98)\end{array}$ & $\begin{array}{c}\text { CMB - } \\
(n=199)\end{array}$ & $\begin{array}{c}\mathrm{CMB}+ \\
(\mathrm{n}=123)\end{array}$ & $\begin{array}{c}\text { CMB - } \\
(n=181)\end{array}$ & $\begin{array}{c}\mathrm{CMB}+ \\
(\mathrm{n}=107)\end{array}$ & $\begin{array}{c}\text { CMB - } \\
(n=325)\end{array}$ \\
\hline Male & $47(48.0)$ & $113(56.8)$ & $73(59.3)$ & $107(59.1)$ & $66(61.7)$ & $217(66.8)$ \\
\hline Age (year) & $70.6[10.4]$ & $69.7[10.3]$ & $66.0[11.0]$ & $63.1[13.0]^{+}$ & $66.7[9.5]$ & $64.6[11.7]$ \\
\hline Hypertension & 74 (75.5) & $134(67.3)$ & 105 (85.4) & $131(72.4)^{+}$ & $98(91.6)$ & $238(73.2)^{+}$ \\
\hline Diabetes Mellitus & $18(18.4)$ & 49 (24.6) & 35 (28.5) & 68 (37.6) & $33(30.8)$ & $131(40.3)$ \\
\hline Hyperlipidemia & $29(29.6)$ & $51(25.6)$ & $72(58.5)$ & $113(62.4)$ & $67(62.6)$ & $177(54.5)$ \\
\hline Coronary artery disease & $11(11.2)$ & $21(10.6)$ & $5(4.1)$ & $10(5.5)$ & $10(9.3)$ & 33 (10.2) \\
\hline Current smoking & $36(36.7)$ & $68(34.2)$ & $50(40.7)$ & $82(45.3)$ & $47(43.9)$ & $156(48.0)$ \\
\hline Previous stroke history & $20(20.4)$ & $43(21.6)$ & $41(33.3)$ & $34(18.8)^{+}$ & $41(38.3)$ & $80(24.9)^{\dagger}$ \\
\hline Use of Antithrombotics & $66(67.3)$ & $110(55.3)$ & $39(31.7)$ & $48(26.5)$ & $48(44.9)$ & 113 (34.8) \\
\hline Antiplatelet agent & $43(43.9)$ & 68 (34.2) & $39(31.7)$ & $48(26.5)$ & $46(43.0)$ & $113(34.8)$ \\
\hline Anticoagulants & $23(23.5)$ & $42(21.1)$ & $0(0.0)$ & $0(0.0)$ & $2(1.9)$ & $0(0.0)$ \\
\hline
\end{tabular}

Results are expressed as a number (\%) or mean [SD].

${ }^{+} P<0.005 ;{ }^{\ddagger} P<0.05$.

differed according to the subtype of ischemic stroke $(P=0.004)$. The presence of any lobar CMB was high in SVO and CE patients, whereas deep CMB was frequently observed from SVO patients (Figure 2). Finally, the ratio of patients with any CMBs in the lobar area to that of deep area was relatively high in patients with CE $(63 / 71 ; 88.7 \%)$ compared to SVO $(71 / 97 ; 73.2 \%)$ or LAA $(43 / 85 ; 50.6 \%)$ cases (Figure 2). The concordance rate between

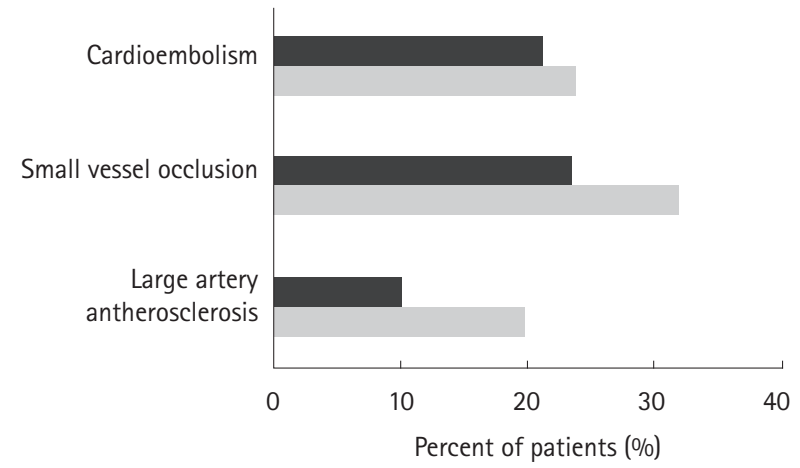


Table 3. Factors associated with the presence of lobar or deep cerebral microbleeds (CMBs)

\begin{tabular}{|c|c|c|c|c|c|c|c|c|}
\hline & \multicolumn{2}{|c|}{ Lobar CMB } & \multicolumn{2}{|c|}{ Any Lobar CMB } & \multicolumn{2}{|c|}{ Deep CMB } & \multicolumn{2}{|c|}{ Any Deep CMB } \\
\hline & OR (95\% Cl) & $P$ & OR (95\% Cl) & $P$ & OR (95\% Cl) & $P$ & OR (95\% Cl) & $P$ \\
\hline Male & $1.03(0.79-1.47)$ & 0.762 & $1.21(0.78-1.87)$ & 0.401 & $0.82(0.58-1.17)$ & 0.275 & $0.50(0.29-0.86)$ & 0.013 \\
\hline Age & $1.01(0.99-1.04)$ & 0.235 & $1.02(1.00-1.04)$ & 0.041 & $1.02(1.00-1.04)$ & 0.013 & $1.00(0.96-1.01)$ & 0.327 \\
\hline Hypertension & $1.14(0.68-1.91)$ & 0.615 & $1.08(0.59-1.96)$ & 0.809 & $1.53(1.03-2.28)$ & 0.035 & $0.84(0.42-1.68)$ & 0.628 \\
\hline Diabetes mellitus & $0.99(0.59-1.65$ & 0.955 & $1.20(0.73-1.96)$ & 0.468 & $0.65(0.43-1.01)$ & 0.053 & $1.13(0.63-2.00)$ & 0.690 \\
\hline Hyperlipidemia & $1.26(0.77-1.70)$ & 0.312 & $0.76(0.49-1.17)$ & 0.213 & $1.10(0.78-1.55)$ & 0.607 & $1.18(0.71-1.98)$ & 0.526 \\
\hline Coronary artery disease & $0.92(0.45-1.89)$ & 0.814 & $0.73(0.33-1.63)$ & 0.445 & $1.04(0.62-1.73)$ & 0.883 & $0.99(0.38-2.56)$ & 0.979 \\
\hline Smoking & $1.15(0.78-1.45)$ & 0.672 & $1.00(0.64-1.55)$ & 0.993 & $1.02(0.71-1.46)$ & 0.909 & $1.12(0.66-1.89)$ & 0.671 \\
\hline Previous stroke history & $1.08(0.62-1.87)$ & 0.789 & $1.16(0.73-1.86)$ & 0.538 & $1.64(1.33-1.81)$ & 0.021 & $1.11(0.63-1.96)$ & 0.707 \\
\hline \multicolumn{9}{|l|}{ Ischemic stroke subtypes } \\
\hline Large artery atherosclerosis & 1 & & 1 & & 1 & & 1 & \\
\hline Small vessel occlusion & $1.74(0.97-3.14)$ & 0.064 & $1.75(1.33-2.16)$ & 0.008 & $1.19(0.80-1.77)$ & 0.401 & $1.91(1.36-2.68)$ & $<0.001$ \\
\hline Cardioembolism & $1.86(1.04-3.34)$ & 0.037 & $2.44(1.60-3.71)$ & $<0.001$ & 0.77 (0.49-1.19) & 0.242 & $1.28(0.90-1.83)$ & 0.174 \\
\hline Use of Antithrombotic agents & $1.81(1.13-2.90)$ & 0.014 & $1.97(1.26-3.07)$ & 0.003 & $1.12(0.76-1.64)$ & 0.712 & $0.93(0.56-1.57)$ & 0.793 \\
\hline
\end{tabular}

$\mathrm{OR}$, odds ratio; $\mathrm{Cl}$, confidence interval.

and previous stroke history were higher in patients with $\mathrm{CMBs}$ than in those without. Patients with CMBs were older (66.0 \pm 11.0 vs. $63.1 \pm 13.0 ; P<0.05)$ than those without $C M B s$ in the SVO group. By multivariate analysis, hypertension $(\mathrm{OR}=1.956$ [95\% confidence interval $=1.064-3.594] ; P=0.031)$ and a previous history of stroke ( $\mathrm{OR}=1.929$ [1.125-3.309]; $P=0.017)$ were independent risk factors for CMBs in SVO patients. Similarly, hypertension $(\mathrm{OR}=3.656[1.760-7.594] ; P=0.001)$ and a previous history of stroke $(\mathrm{OR}=1.663[1.036-2.669] P=0.035)$ were also independent risk factors for CMBs in LAA patients. However, these factors were not associated with the presence of CMBs in CE patients.

\section{Risk factors of CMB according to location}

In terms of CMB location, the results of univariable analysis are demonstrated at Table 3. According to the results of multivariable analysis, the presence of strictly lobar $\mathrm{CMB}$ was independently associated with $\mathrm{CE}(\mathrm{OR}=1.85[1.02-3.34] ; P=0.042)$ and use of antithrombotic agents $(\mathrm{OR}=1.80$ [1.10-2.94]; $P=$ $0.019)$. Age $(\mathrm{OR}=1.02[1.00-1.04] ; P=0.015)$ and hypertension $(\mathrm{OR}=1.61[1.08-2.40] ; P=0.020)$ were independently associated with the presence of deep CMB. The presence of any lobar CMB was independently associated with the use of antithrombotic agents $(\mathrm{OR}=1.97[1.26-3.07] ; P=0.003)$, whereas sex was the only factor associated with the presence of any deep CMB (male sex: $\mathrm{OR}=0.50[0.29-0.86] ; P=0.013)$.

\section{Discussion}

Our present findings indicate that the prevalence, location, and risk factors associated with CMBs differ significantly according to ischemic stroke subtype. CMBs were most frequently ob- served in SVO patients, followed by CE and LAA patients. The distribution of CMBs was mostly in deep areas in SVO and LAA patients, whereas the CMBs were found in the lobar area relatively more frequently in CE patients compared to SVO and LAA patients. The presence of CMBs was associated with hypertension and previous stroke history in SVO or LAA cases, whereas there was no significant association between those factors and the presence of CMBs in patients with CE. Prior use of antithrombotics was associated with the presence of any lobar CMB, which was relatively frequent in CE patients.

The prevalence of CMB according to ischemic stroke subtype in our current series was consistent with the findings of previous reports. Not only the prevalence, but also the CMB location, differed according to ischemic stroke subtype. SVO patients more frequently demonstrated CMBs in deep areas, where the deep perforating arteries exist. ${ }^{19,20}$ Previously, CMBs located in deep areas were found to be associated with high blood pressure, renal impairment, and heavy alcohol consumption. ${ }^{21-23}$ From our present study findings, CMBs in SVO patients were associated with hypertension and a previous history of stroke. CMBs in those areas are suggested to be caused by hypertensive arteriopathy, which affects the perforators. ${ }^{24}$

CMBs in lobar areas have been associated with smoking, APOE $\varepsilon 4$, and low cholesterol levels. ${ }^{5,22}$ Lobar CMBs are also regarded as an imaging biomarker for cerebral amyloid angiopathy. ${ }^{25}$ Recently, several studies revealed lobar CMBs in specific lobar areas to be associated with various degenerative diseases (e.g. Alzheimer's disease, Parkinson's disease and Diffuse Lewy body disease). ${ }^{26-28}$ However, age, which is the strongest risk factor for degenerative diseases, was not found to be associated with lobar CMBs in our present study with stroke population. Instead, we found that $C E$ and the previous use of antithrombotics were as- 
sociated with the presence of lobar CMB.

CMBs have previously been associated with the use of anticoagulants, and this relationship was reported to be stronger in patients from the general population with a greater fluctuation of prothrombin time. ${ }^{29}$ Furthermore, among patients with ischemic stroke, CMBs were found to be common in patients with atrial fibrillation, and the use of antithrombotics was independently associated with the presence of lobar but not deep CMBs. ${ }^{13}$ In our present study, lobar CMBs were more frequent in CE patients, and the presence of lobar CMB was associated with a prior use of antithrombotics. Patients with CE generally show small asymptomatic cortical infarctions, higher spontaneous recanalization rates, and concomitant hemorrhagic transformations. ${ }^{30,31}$ Considering that patients under oral anticoagulation are more likely to develop CMBs, the use of antithrombotics may enhance micro-hemorrhages occurring as a result of recanalization of small arterioles obstructed by an asymptomatic embolus originating from the heart. ${ }^{32-34}$ The characteristics of lobar CMBs developed through such a mechanism may differ from CMBs caused by degenerative disease.

Our study has a number of limitations of note. First, due to its retrospective design, there was a risk of selection bias, which could have impacted on our findings of cause and effect. However, our patients were consecutively recruited from a single center, and most of these cases underwent brain MRI using an established protocol. Second, we used both 1.5 T and 3.0 T MRI, although 1.5 T MRI is inferior for the detection of CMBs. ${ }^{35}$ Thus, the prevalence of CMBs may have been underestimated. Furthermore, we used $5 \mathrm{~mm}$ as the maximum diameter for defining $\mathrm{CMB}$. This may also have influenced the prevalence of CMBs to be underestimated.

\section{Conclusions}

Notwithstanding the above limitations, our present study demonstrated that the distribution and risk factors associated with CMBs differed by stroke subtype, especially in patients with CE compared with SVO or LAA. Our results suggest different pathogenic mechanisms for the development of CMBs of each subtype and that the previous use of antithrombotic agents is related to $\mathrm{CMB}$ location.

\section{References}

1. Schrag M, McAuley G, Pomakian J, Jiffry A, Tung S, Mueller C, et al. Correlation of hypointensities in susceptibility-weighted images to tissue histology in dementia patients with cerebral amyloid angiopathy: a postmortem MRI study. Acta Neuro- pathol 2010;119:291-302.

2. Wardlaw JM, Smith EE, Biessels GJ, Cordonnier C, Fazekas F, Frayne $R$, et al. Neuroimaging standards for research into small vessel disease and its contribution to ageing and neurodegeneration. Lancet Neurol 2013;12:822-838.

3. Kim BJ, Lee SH. Cerebral microbleeds: their associated factors, radiologic findings, and clinical implications. J Stroke 2013;15: 153-163.

4. Norrving B. Evolving concept of small vessel disease through advanced brain imaging. J Stroke 2015;17:94-100.

5. Poels MM, Vernooij MW, Ikram MA, Hofman A, Krestin GP, van $\operatorname{der}$ Lugt $A$, et al. Prevalence and risk factors of cerebral microbleeds: an update of the Rotterdam scan study. Stroke 2010; 41(10 Suppl):S103-S106.

6. Vernooij MW, van der Lugt A, Ikram MA, Wielopolski PA, Niessen WJ, Hofman A, et al. Prevalence and risk factors of cerebral microbleeds: the Rotterdam Scan Study. Neurology 2008; 70:1208-1214.

7. Gregoire SM, Scheffler G, Jäger HR, Yousry TA, Brown MM, Kallis $C_{1}$ et al. Strictly lobar microbleeds are associated with executive impairment in patients with ischemic stroke or transient ischemic attack. Stroke 2013:44:1267-1272.

8. Klein I, lung B, Labreuche J, Hess A, Wolff M, Messika-Zeitoun $D$, et al. Cerebral microbleeds are frequent in infective endocarditis: a case-control study. Stroke 2009;40:3461-3465.

9. Greenberg SM, Vernooij MW, Cordonnier C, Viswanathan A, Al-Shahi Salman R, Warach S, et al. Cerebral microbleeds: a guide to detection and interpretation. Lancet Neurol 2009;8: 165-174.

10. Werring DJ, Frazer DW, Coward $\sqcup$, Losseff NA, Watt H, Cipolotti $L$, et al. Cognitive dysfunction in patients with cerebral microbleeds on T2*-weighted gradient-echo MRI. Brain 2004; 127(Pt 10):2265-2275.

11. Koennecke HC. Cerebral microbleeds on MRI: prevalence, associations, and potential clinical implications. Neurology 2006; 66:165-171.

12. Saito T, Kawamura Y, Tanabe Y, Asanome A, Takahashi K, Sawada $J$, et al. Cerebral microbleeds and asymptomatic cerebral infarctions in patients with atrial fibrillation. J Stroke Cerebrovasc Dis 2014;23:1616-1622.

13. Horstmann $S$, Möhlenbruch $M$, Wegele $C$, Rizos $T$, Laible $M$, Rauch $\mathrm{G}$, et al. Prevalence of atrial fibrillation and association of previous antithrombotic treatment in patients with cerebral microbleeds. Eur J Neurol 2015;22:1355-1362.

14. Chatzikonstantinou A, Willmann O, Szabo K, Hennerici MG. Cerebral microbleeds are uncommon in ischemic stroke associated with nonvalvular atrial fibrillation. J Neuroimaging 2011; 21:103-107. 
15. Kato $H_{1}$ Izumiyama $M$, Izumiyama $K$, Takahashi $A$, Itoyama $Y$. Silent cerebral microbleeds on $\mathrm{T}^{*}$-weighted MRI: correlation with stroke subtype, stroke recurrence, and leukoaraiosis. Stroke 2002;33:1536-1540.

16. Naka $H$, Nomura $E$, Wakabayashi $S$, Kajikawa $H$, Kohriyama $T_{1}$ Mimori $Y$, et al. Frequency of asymptomatic microbleeds on T2*-weighted MR images of patients with recurrent stroke: association with combination of stroke subtypes and leukoaraiosis. AJNR Am J Neuroradiol 2004;25:714-719.

17. Adams HP Jr, Bendixen BH, Kappelle U, Biller J, Love BB, Gordon $\mathrm{DL}$, et al. Classification of subtype of acute ischemic stroke. Definitions for use in a multicenter clinical trial. TOAST. Trial of Org 10172 in Acute Stroke Treatment. Stroke 1993;24:35-41.

18. Furie KL, Kasner SE, Adams RJ, Albers GW, Bush RL, Fagan SC, et al. Guidelines for the prevention of stroke in patients with stroke or transient ischemic attack: a guideline for healthcare professionals from the American Heart Association/American Stroke Association. Stroke 2011;42:227-276.

19. Gregoire SM, Jäger HR, Yousry TA, Kallis C, Brown MM, Werring DJ. Brain microbleeds as a potential risk factor for antiplatelet-related intracerebral haemorrhage: hospital-based, case-control study. J Neurol Neurosurg Psychiatry 2010;81: 679-684.

20. Sun J, Soo YO, Lam WW, Wong KS, Zeng JS, Fan YH. Different distribution patterns of cerebral microbleeds in acute ischemic stroke patients with and without hypertension. Eur Neurol 2009;62:298-303.

21. Zhang $C$, Li Z, Wang Y, Zhao X, Wang C, Liu L, et al. Risk factors of cerebral microbleeds in strictly deep or lobar brain regions differed. J Stroke Cerebrovasc Dis 2015;24:24-30.

22. Ding J, Sigurdsson S, Garcia M, Phillips CL, Eiriksdottir G, Gudnason $\mathrm{V}$, et al. Risk factors associated with incident cerebral microbleeds according to location in older people: the Age, Gene/Environment Susceptibility (AGES)-Reykjavik Study. JAMA Neurol 2015;72:682-688.

23. Song TJ, Kim J, Lee HS, Nam CM, Nam HS, Kim YD, et al. Distribution of cerebral microbleeds determines their association with impaired kidney function. J Clin Neurol 2014;10:222-228.

24. Werring DJ. Cerebral microbleeds: clinical and pathophysiological significance. J Neuroimaging 2007;17:193-203.
25. Viswanathan A, Greenberg SM. Cerebral amyloid angiopathy in the elderly. Ann Neurol 2011;70:871-880.

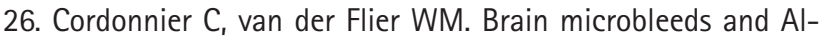
zheimer's disease: innocent observation or key player? Brain 2011;134(Pt 2):335-344.

27. Gungor I, Sarro L, Graff-Radford J, Zuk SM, Tosakulwong N, Przybelski SA, et al. Frequency and topography of cerebral microbleeds in dementia with Lewy bodies compared to Alzheimer's disease. Parkinsonism Relat Disord 2015;21:11011104.

28. Yamashiro $K$, Tanaka $R$, Hoshino $Y$, Hatano T, Nishioka K, Hattori $\mathrm{N}$. The prevalence and risk factors of cerebral microbleeds in patients with Parkinson's disease. Parkinsonism Relat Disord 2015;21:1076-1081.

29. Akoudad S, Darweesh SK, Leening MJ, Koudstaal PJ, Hofman $A$, van der Lugt $A$, et al. Use of coumarin anticoagulants and cerebral microbleeds in the general population. Stroke 2014; 45:3436-3439.

30. Koennecke HC, Bernarding J, Braun J, Faulstich A, Hofmeister $C$, Nohr $R$, et al. Scattered brain infarct pattern on diffusionweighted magnetic resonance imaging in patients with acute ischemic stroke. Cerebrovasc Dis 2001;11:157-163.

31. Mast H, Thompson JL, Völler H, Mohr JP, Marx P. Cardiac sources of embolism in patients with pial artery infarcts and lacunar lesions. Stroke 1994;25:776-781.

32. Fisher M, French $S$, Ji $P$, Kim RC. Cerebral microbleeds in the elderly: a pathological analysis. Stroke 2010;41:2782-2785.

33. Gregoire SM, Werring DJ, Chaudhary UJ, Thornton JS, Brown MM, Yousry TA, et al. Choice of echo time on GRE T2*-weighted MRI influences the classification of brain microbleeds. Clin Radiol 2010;65:391-394.

34. Vernooij MW, Haag MD, van der Lugt A, Hofman A, Krestin GP, Stricker $\mathrm{BH}$, et al. Use of antithrombotic drugs and the presence of cerebral microbleeds: the Rotterdam Scan Study. Arch Neurol 2009;66:714-720.

35. Scheid R, Ott DV, Roth $H$, Schroeter ML, von Cramon DY. Comparative magnetic resonance imaging at 1.5 and 3 Tesla for the evaluation of traumatic microbleeds. J Neurotrauma 2007;24: 1811-1816. 